\title{
Asymptotic freedom in massive Yang-Mills theory
}

\author{
J. Gegelia ${ }^{1}$ \\ ${ }^{1}$ Institut für Kernphysik, Johannes Gutenberg-Universität, J.J. Becherweg 45, \\ D-55099 Mainz, Germany \\ and \\ High Energy Physics Institute of TSU, Tbilisi, Georgia
}

(Dated: December 12, 2018)

\begin{abstract}
An effective field theory model of the massive Yang-Mills theory is considered. Assuming that the renormalized coupling constants of 'non-renormalizable' interactions are suppressed by a large scale parameter it is shown that in analogy to the non-abelian gauge invariant theory the dimensionless coupling constant vanishes logarithmically for large values of the renormalization scale parameter.
\end{abstract}

PACS numbers: 11.10.Gh, 03.70.+k, 11.10.Hi

The standard model (SM) is an established consistent theory of strong, electromagnetic and weak interactions. It describes most of the known phenomena in elementary particle physics. The modern point of view is to think of the SM as an effective field theory, "low-energy approximation to a deeper theory that may not even be a field theory, but something different like a string theory" [1]. While the effective Lagrangian consists of an infinite number of terms, the non-renormalizable interactions are suppressed by powers of a large scale. This makes the contributions of these interactions negligible for energies much lower than the large scale. Renormalizability in the traditional sense is not considered as a fundamental principle but rather a property of the leading order approximation to the full effective theory.

The SM takes gauge invariance as a starting point. In modern string theories [2] one first notices a state of mass zero and unit spin among the normal modes of a string, and then from that deduces the gauge invariance of the effective field theory that describes such particles [1]. In modern approach one usually takes gauge invariance as the starting point. Referring again to Weinberg's book, "It is too soon to tell which of these two alternatives corresponds to the logical order of nature" [1]. Taking into account the above considerations it is not evident that the gauge invariance principle should be applied to weak interaction as it is not mediated by massless vectors. As the fundamental nature of both the gauge invariance and the renormalizability seems to be questionable, the role of the scalar sector of the electro-weak theory could be also questioned, especially as the experimental status of the scalar Higgs particle remains still unclear. Therefore it seems interesting to have a closer look to effective field theory models of massive vector mesons which are not based on the concept of gauge invariance.

In the present work an effective field theory model of the massive vector mesons interacting with fermions is considered. I do not deal here with the most general effective Lagrangian, but instead add the mass term of the vector mesons to the standard QCD Lagrangian [3]. Next I add an infinite number of non-renormalizable interactions which are required to absorb the divergences generated by loop diagrams of the perturbation theory. I assume that all coupling constants of non-renormalizable interactions are suppressed by powers of a Large scale parameter $\Lambda$. Under this assumption I study the renormalization group behavior of the dimensionless coupling constant to one-loop order. The aim of this work is to investigate if the asymptotic freedom of the non-abelian gauge theories [4, 5] persist when the explicit mass term is introduced in the Lagrangian (For earlier works on asymptotically free massive Yang-Mills models see Refs. [6, 7]). Notations below closely follow the book by Ynduráin [3].

Let us consider an EFT model described by the following bare Lagrangian:

$$
\mathcal{L}=\mathcal{L}_{\mathcal{Q C D}}+\frac{M^{2}}{2} \sum_{b} B_{a}^{\mu} B_{a \mu}+\mathcal{L}_{1}
$$

where

$$
\mathcal{L}_{\mathcal{Q C D}}=\sum_{q} \sum_{j} \bar{q}^{j}\left(i \not \partial-m_{q}\right) q^{j}+g \sum_{q} \sum_{i k a} \bar{q}^{i} \gamma_{\mu} t_{i k}^{a} q^{k} B_{a}^{\mu}-\frac{1}{4} \sum_{a} G_{a}^{\mu \nu} G_{a \mu \nu}
$$

is the standard QCD Lagrangian [3] and $\mathcal{L}_{1}$ contains an infinite number of terms of non-renormalizable interactions which are necessary for the cancelation of divergences of loop diagrams.

Let us investigate the renormalization group behavior of renormalized coupling constant $g_{R}$ by analyzing the $B \bar{\psi} \psi$ Green's function using dimensional regularization (with parameter $n$ ) in combination with the MS scheme.

To carry out the renormalization let us introduce the renormalized quantities

$$
q^{j}=Z_{q}^{1 / 2} q_{R}^{j}, B_{a}^{\mu}=Z_{B}^{1 / 2} B_{a, R}^{\mu}, m_{q}=Z_{m}^{-1} m_{q, R}, M^{2}=Z_{M}^{-1} M_{R}^{2}, g=Z_{g}^{-1} g_{R}, \cdots,
$$


(where $\cdots$ stands for parameters hidden in $\mathcal{L}_{1}$ ) and choose $Z_{q}, Z_{B}, Z_{m}, Z_{M}, Z_{g}, \cdots$ such that all Green's functions are finite order-by-order in loop expansion.

The sum of one-particle irreducible diagrams contributing in vector-meson propagator is parameterized as $-i \Pi^{\mu \nu}$ and to one loop order consists of a fermion and vector meson contributions.

Divergent part of the contribution of all $n_{f}$ fermions reads:

$$
\Pi_{F ; a b}^{\mu \nu}=-2 T_{F} \delta_{a b} \frac{g_{R}^{2}}{16 \pi^{2}}\left(-g^{\mu \nu} q^{2}+q^{\mu} q^{\nu}\right) \frac{2}{3} N_{\epsilon} n_{f}
$$

where $N_{\epsilon}=2 /(4-n)$ and $T_{F}=1 / 2$. This has to be canceled by counter-term contribution generated by $Z_{B}$.

Divergent part of the contribution of vector fields is given as

$$
\Pi_{a b ; g}^{\mu \nu}=\frac{C_{A} \delta_{a b} g_{R}^{2}}{32 \pi^{2}} N_{\epsilon}\left[-9 M^{2} g^{\mu \nu}+7\left(q^{\mu} q^{\nu}-g^{\mu \nu} q^{2}\right)-\frac{\left(q^{\mu} q^{\nu}-g^{\mu \nu} q^{2}\right)\left(14 M^{2} q^{2}+q^{4}\right)}{12 M^{4}}\right]
$$

The first term in square brackets in Eq. (5) is canceled by mass counter-term, second is canceled by $Z_{B}$ and the third term is taken care by counter-term contributions generated by higher-order terms hidden in $\mathcal{L}_{1}$.

Field redefinition constant obtained from Eqs. (4) and (5) reads

$$
Z_{B}=1+\frac{g_{R}^{2}}{16 \pi^{2}} N_{\epsilon}\left[\frac{7 C_{A}}{2}-\frac{4 T_{F} n_{f}}{3}\right]
$$

The sum of one-particle irreducible diagrams contributing in fermion propagator is given by

$$
\Sigma_{j i}^{d i v}(p)=\frac{g_{R}^{2}}{16 \pi^{2}} \mu^{4-n} C_{F} \delta_{j i} N_{\epsilon}\left[3 m-\frac{m^{3}}{M^{2}}+\frac{3 m^{2}}{2 M^{2}} \not p-\frac{p^{2}}{2 M^{2}} \not p\right] .
$$

The first two terms in square brackets in Eq. (7) are canceled by mass counter-term, third is canceled by $Z_{q}$ and the fourth term is taken care by counter-term contributions generated by higher-order terms hidden in $\mathcal{L}_{1}$.

Field redefinition constant obtained from Eq. (17) reads

$$
Z_{q}=1+\frac{3 m^{2} g_{R}^{2}}{32 \pi^{2} M^{2}} C_{F} N_{\epsilon}
$$

This gives the corresponding counter-term diagram contribution in $B \bar{\psi} \psi$ vertex function

$$
i \Gamma_{i j a}^{(1) \mu, Z_{q}}=\frac{3 i g_{R}^{3} m^{2}}{32 \pi^{2} M^{2}} C_{F} t_{i j}^{a} N_{\epsilon} \mu^{4-n} \gamma^{\mu} .
$$

Two one-loop diagrams contribute in $B \bar{\psi} \psi$ vertex function. Keeping only momentum independent divergent part ${ }^{1}$ contributing in the renormalization of $g$ the first diagram gives

$$
i \Gamma_{i j a}^{(1) \mu}=-\frac{3 i}{64 \pi^{2}} \frac{m^{2}}{M^{2}} g_{R}^{3} C_{A} t_{j i}^{a} \mu^{4-n} N_{\epsilon} \gamma^{\mu} .
$$

Analogous divergent part of the second diagram reads

$$
i \Gamma_{i j a}^{(2) \mu}=-\frac{3 i}{32 \pi^{2}} \frac{m^{2}}{M^{2}} g_{R}^{3}\left(C_{F}-\frac{1}{2} C_{A}\right) t_{j i}^{a} \mu^{4-n} N_{\epsilon} \gamma^{\mu} .
$$

For the momentum-independent divergent part of the sum of two diagrams we obtain

$$
i \Gamma_{i j a}^{(1) \mu}+i \Gamma_{i j a}^{(2) \mu}=-\frac{3 i g_{R}^{3} m^{2}}{32 \pi^{2} M^{2}} C_{F} t_{i j}^{a} N_{\epsilon} \mu^{4-n} \gamma^{\mu} .
$$

The contributions of two loop diagrams, Eq. (12), is exactly canceled by counter-term diagram contribution, Eq. (9), and the resulting value for $Z_{g}$ at one-loop order is

$$
Z_{g}=Z_{B}^{-1 / 2}=1-\frac{g_{R}^{2}}{32 \pi^{2}} N_{\epsilon}\left[\frac{7 C_{A}}{2}-\frac{4 T_{F} n_{f}}{3}\right]
$$

\footnotetext{
1 The momentum dependent divergent parts are canceled by counter-term contributions generated by corresponding terms in $\mathcal{L}_{1}$.
} 
For the corresponding running coupling constant $\alpha=g_{R}^{2} /(4 \pi)$ one obtains

$$
\alpha(\mu)=\frac{\alpha\left(\mu_{0}\right)}{1+\frac{\alpha\left(\mu_{0}\right)}{2 \pi} \ln \frac{\mu}{\mu_{0}}\left[\frac{7 C_{A}}{2}-\frac{4 T_{F} n_{f}}{3}\right]} .
$$

The renormalized coupling $\alpha$ of Eq. (14) vanishes logarithmically for large $\mu$ if $n_{f}<21 C_{A} / 4$.

To make sure that the considered model is self-consistent, I have checked that the renormalization of the $B B B$ Green's functions leads to the same results as given in Eqs. (13)-(14).

To conclude, renormalization group behavior of the dimensionless coupling constant of an effective field theory model of massive Yang-Mills vector fields interacting with fermions has been considered. This analysis has been performed under assumption that the coupling constants of non-renormalizable interactions are suppressed by powers of a large scale parameter $\Lambda$. The renormalized coupling constant vanishes logarithmically for large (but still $<<\Lambda$ ) values of the renormalization scale parameter $\mu$ if the number of fermions $n_{f}<21 C_{A} / 4$. This is to be compared with $n_{f}<11 C_{A} / 2$ in the gauge invariant theory. For $\mu \sim \Lambda$ the contributions of the non-renormalizable interactions can not be neglected, therefore Eq. (14) can not be trusted for such values of $\mu$. The same applies to QCD as well. According to modern understanding QCD should be considered as the leading order approximation to an effective field theory. For the energies where the contributions of non-renormalizable interactions are no longer suppressed, the asymptotic freedom of the strong interaction described by standard renormalizable QCD Lagrangian requires further investigation. Of course it is not evident that at such high energies the quantum field theory approach is still meaningful.

\section{Acknowledgments}

The author would like to thank G. Gabadadze for comments on the manuscript. This work was supported by the Deutsche Forschungsgemeinschaft (SFB 443).

[1] S. Weinberg, "The Quantum Theory Of Fields. Vol. 1: Foundations," Cambridge, UK: Univ. Pr. (1995).

[2] M. B. Green, J. H. Schwarz and E. Witten, "Superstring Theory". Cambridge, Uk: Univ. Pr. ( 1987). ( Cambridge Monographs On Mathematical Physics): Section 2.2.

[3] F. J. Yndurain, "The theory of quark and gluon interactions," Berlin, Germany: Springer (1999).

[4] H. D. Politzer, Phys. Rev. Lett. 30, 1346 (1973).

[5] D. J. Gross and F. Wilczek, Phys. Rev. D 8, 3633 (1973).

[6] J. P. Hsu, Lett. Nuovo Cim. 11, 129 (1974).

[7] W. A. Bardeen and K. i. Shizuya, Phys. Rev. D 18, 1969 (1978). 